\title{
Evidence of a humoral response to a novel protein WARF4 embedded in the West Nile virus NS4B gene encoded by an alternative open reading frame
}

\author{
GIOVANNI FAGGIONI ${ }^{1}$, ANDREA CIAMMARUCONI ${ }^{1}$, RICCARDO DE SANTIS ${ }^{1}$, \\ ALICE POMPONI $^{1}$, MARIA TERESA SCICLUNA ${ }^{2}$, KATIA BARBARO ${ }^{2}$, LAURA MASUELLI ${ }^{3}$, \\ GIANLUCA AUTORINO ${ }^{2}$, ROBERTO BEI ${ }^{4}$ and FLORIGIO LISTA ${ }^{1}$
}

\begin{abstract}
${ }^{1}$ Laboratory of Molecular Biology, Army Medical Research Centre, I-00184 Rome; ${ }^{2}$ National Reference Centre for Equine Diseases-Istituto Zooprofilattico Sperimentale delle Regioni Lazio e Toscana, I-00178 Rome; ${ }^{3}$ Department of Experimental Medicine, University of Rome Sapienza, I-00161 Rome; ${ }^{4}$ Department of Experimental Medicine and Biochemical Sciences, University of Rome 'Tor Vergata' I-00133 Rome, Italy
\end{abstract}

Received September 30, 2008; Accepted November 17, 2008

DOI: $10.3892 /$ ijmm_00000158

\begin{abstract}
West Nile virus (WNV) is a flavivirus that is maintained in a bird-mosquito transmission cycle. Humans, horses and other non-avian vertebrates are usually incidental hosts. However, WNV is a neurotropic virus, which requires an efficient humoral response for the control of a neuroinvasive infection. The WNV genome encodes three structural (capsid, premembrane/membrane and envelope) and seven nonstructural proteins. Bioinformatic analysis performed on the WNV genomes detected a conserved alternative open reading frame restricted to the lineage I virus. To quickly verify the existence of this putative protein, entitled West Nile Alternative Reading Frame 4 (WARF4), we produced a prokaryotic recombinant source of WARF4 and verified its immunogenicity in vivo by analyzing 43 horse serum samples, of which 15 were positive for antibodies to WNV premembrane and envelope (prM-E) proteins. Specific antibodies to WARF4 were significantly detected in 5 out of the 15 serum samples testing positive for antibodies to prM-E WNV proteins. Our findings provide evidence of a significant antibody response to the WARF4 protein in the serum of the horse testing positive for antibodies to prM-E proteins, thus indicating that this antigen might be a potential tool for further characterization of the immune response of WNV infections in humans as well.
\end{abstract}

Correspondence to: Dr Florigio Lista, Health Corps, Italian Army, Head, Histology and Molecular Biology Section, Army Medical and Veterinary Research Center, Via Santo Stefano Rotondo 4, 00184 Roma, Italy

E-mail: florigio.lista@esercito.difesa.it

Key words: West Nile virus, antibodies, alternative open reading frame, west nile alternative reading frame 4

\section{Introduction}

Following its original isolation, West Nile virus (WNV) was implicated in sporadic outbreaks of mild viral illness in Africa, the Middle East, western Asia and Europe $(1,2)$. Since 1999 , the virus has emerged as a new threat to humans and mammals in the western hemisphere $(3,4)$. Phylogenetic analyses reveal two distinct lineages (5): lineage I, which has a worldwide distribution and is involved in human and equine outbreaks, and lineage II, which is present in Africa's subSaharan region and is not associated with clinical manifestations in humans $(6,7)$. Although several studies are in progress for a better understanding of WNV pathogenesis, the mechanisms of immunological escape of West Nile remain unclear (8). Moreover, it is suggested that unidentified factors may be involved in the West Nile disease (9). WNV can be transmitted to humans and can cause encephalitis, depending on age and the immunity status of the exposed individual (3). Antibody-positive blood components are less efficient transmitters of the virus than antibody-negative components, thus indicating that neutralizing antibodies are essential for the control of WNV infection in vivo and that an efficient humoral response is critical for the control of a neuroinvasive WNV infection $(10,11)$.

To obtain a major number of proteins without modifying the genome size, viruses often make use of an alternative reading frame gene embedded in the mean coding strand. The existence of superimposed genes, first noted in bacteriophage $\phi \mathrm{X} 174$ (12), is a phenomenon well characterized in viruses as well as in other organisms (13-15). Among the flavivirdae, the use of an alternative open reading frame protein (ARFP) has been reported for hepatitis C viruses (HCV) (16). After humans, horses constitute the majority of mammals infected with WNV (17). The close phylogenetic relationship between $\mathrm{HCV}$ and West Nile led us to analyze the WNV genome, searching for alternative gene products. Our bioinformatic analysis detected six ARFPs, one of them restricted to lineage I of WNV. To quickly verify the existence of this putative 

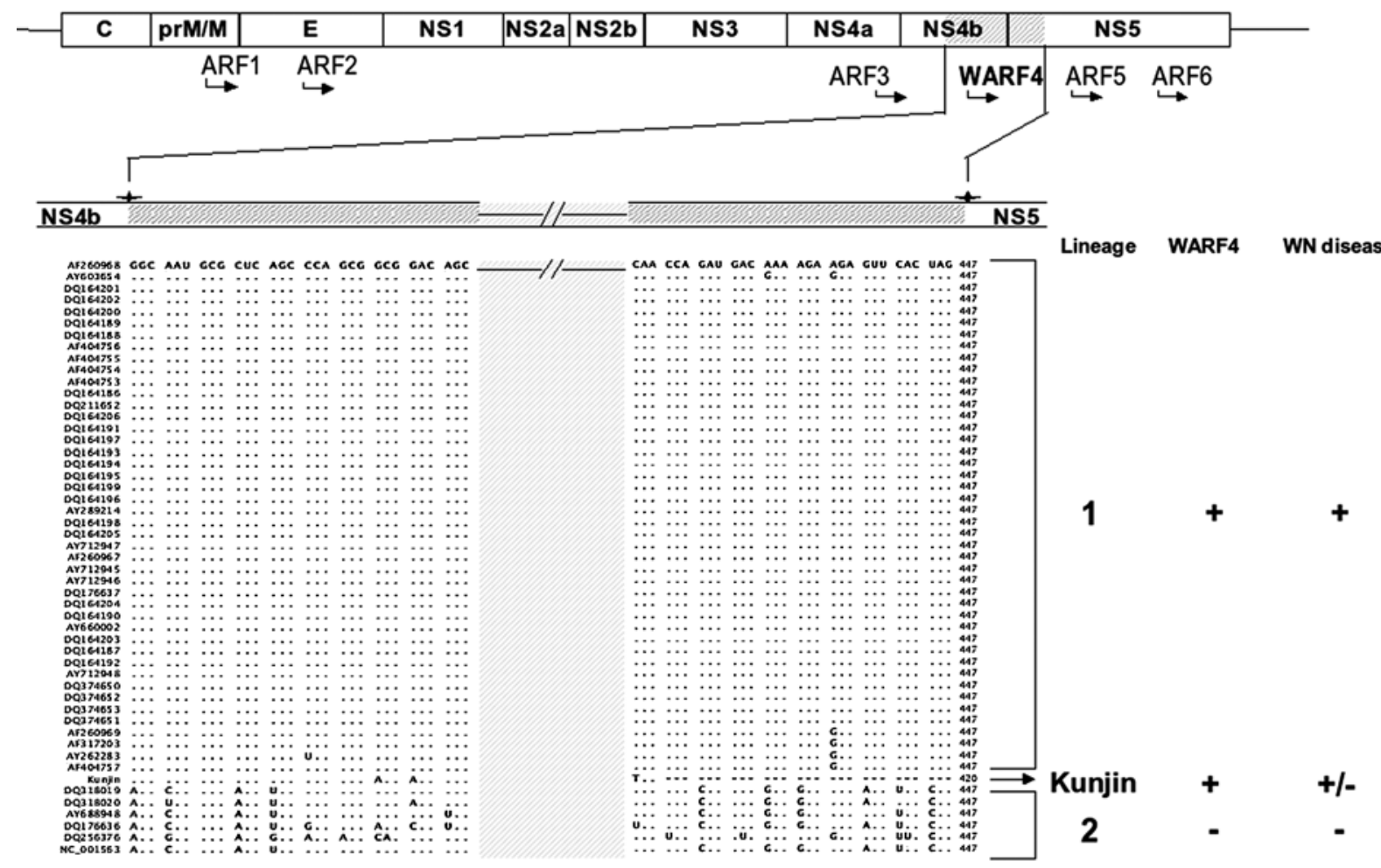

Figure 1. Schematic organization of the West Nile genome and positions of the alternative reading frames. Illustrated in detail are the partial alignment of WARF4 and its association with viral lineage and disease.

protein, entitled West Nile Alternative Reading Frame 4 (WARF4), we produced a prokaryotic recombinant source of WARF4 and tested its immunogenicity in vivo by analyzing 43 horse serum samples, of which 15 were positive for antibodies to WNV premembrane and envelope (prM-E) proteins. Our findings provide evidence of a significant antibody response to the WARF4 protein in the prM-E protein positive horse, thus indicating that this antigen might be a potential tool for further characterization of the immune response to WNV infections in humans as well.

\section{Materials and methods}

Bioinformatic analysis. Fifty West Nile viral genomes (Fig. 1) were retrieved from GenBank. ARFs were detected by CLC Sequence Viewer (www.clcbio.com). The search parameters included any codon used as start codon and an ARF protein length of at least 100 amino acids including a stop codon. Further analyses were carried out using ClustalX (http://www. clustal.org/).

Serum samples and genomic RNA. Forty-three horse serum samples were collected by Istituto Zooprofilattico Sperimentale delle Regioni Lazio e Toscana during the serological surveillance activities conducted in Toscana (Italy) between 1998 and 2007. The sera were tested according to the manufacturer's instructions for anti-West Nile IgG using the commercial Kit ELISA ID Screen ${ }^{\circledR}$ West Nile Competition (ID.VET, Montpellier-France). West Nile genome RNA was obtained from the European Network for Diagnostics of Imported Viral Disease (ENIVD) during the External Quality Assurance (EQA) for molecular detection of
West Nile virus. The nucleic acids were extracted using the NucleoSpin RNA Virus Kit according to the manufacturer's instructions (Macherey-Nagel, Düren, Germany).

Cloning and espression of WARF4 protein. The fragment of 394 bp spanning the WARF4 position 7327-7720 (accession number AF260967) was amplified in a single step RT-PCR using 5' forward oligonucleotide (gttgaggaattctttccaaacctct cccaag) and 3' reverse oligonucleotide (atgcgcggatcccagc ggcggacagcggctgg). The fragment was cloned into the expression vector pRSETC and then expressed in the BL21 Star (DE3)pLysS competent cells (Invitrogen, CA). The histagged recombinant protein (WARF4) was purified under denaturing condition with Ni-NTA Agarose resin (Qiagen, Germany). Proteins were visualized by Coomassie Blue staining.

Western blot analysis. Approximately $200 \mathrm{ng}$ of the WARF4 protein or the bacterial cell extract transformed with the plasmid vector alone was separated by SDS/PAGE and then transferred to a nitrocellulose membrane. After blocking, membranes were incubated with horse serum (1:1000) overnight at $4^{\circ} \mathrm{C}$. The $1: 1000$ dilution was chosen for testing as the highest serum concentration that lacked substantial background reactivity. After washing, the filters were incubated with anti-horse $\operatorname{IgG}$ whole-molecule peroxidase conjugate (Sigma) and developed by a chemiluminescent kit (Sigma) as previously described (18-20). Criteria of positivity was the appearance of an immunoreactive band in the WARF4 sample, co-migrating with the one visualized by the anti-his antibody and not in the bacterial cell extract used as negative control. 


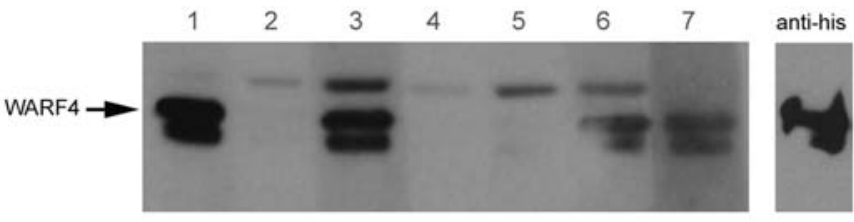

Figure 2. Immunoreactivity of horse sera to the WARF4 recombinant protein. Seven prM-E protein positive horse serum samples assayed for humoral response to the recombinant protein WARF4 are shown. Four serum samples $(1,3,6,7)$ specifically detected the WARF4 protein by Western blotting. Reactivity with the anti-his antibody to WARF4 recombinant protein is shown as the positive control.

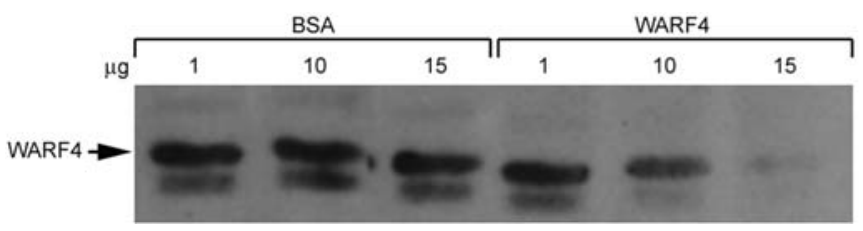

Figure 3. Specificity of the anti-WARF4 antibodies. The analysis was performed to confirm the specificity of the serum antibodies to WARF4 protein by a competition assay. One WARF4 positive horse serum sample preincubated with increasing amounts of BSA as negative control (lanes 1, $2,3$ ) and WARF4-free antigen (lanes $4,5,6)$ is shown. The samples were then analyzed by Western blotting.

Competition assay. Competition assay was performed to confirm the specificity of the serum IgG for the WARF4 protein. Increasing concentrations of the WARF4 purified protein or bovine serum albumin (BSA) (1-15 $\mu \mathrm{g})$ were incubated for $30 \mathrm{~min}$ at room temperature with horse serum testing positive for antibodies to WARF4 protein. The serum was then tested for its reactivity with WARF4 by Western blotting as described above.

Statistical analysis. Associations were considered significant at $\mathrm{p}$-values $\leq 0.05$ by a $2 \times 2$ contingency table.

\section{Results}

In silico analysis detected six ARFs (Fig. 1). Among the others, the ARF4 appears to be the longest (447 bp) and most conserved from the genomes, present in $88 \%$ (44/50). This novel alternative reading frame, entitled WARF4, begins from $\mathrm{a}+2$ position when compared to the coding strand. This novel potential gene is embedded between the NS4b Cterminal residue and the NS5 N-terminal residue (73117757 bp, accession number AF260967), starting from a GGC triplet and terminating with a TAG stop codon. The WARF4 amino acid sequence does not show an apparent homology with other known protein sequences. Assuming that the initiation codon is the first in the alternative frame, the protein would contain $148 \mathrm{AA}$ and shows an isoelectric point of 9.4. Bioinformatic analysis predicts a myristoylation signal in position 1 to 10 AA with a low probability of false-positive prediction. In order to prove the actual existence of the WARF4 protein, we produced a prokaryotic recombinant source of WARF4 and verified its immunogenicity in vivo by
Table I. Evidence of antibodies to WARF4 protein in horse sera testing positive for prM-E WNV proteins.

$\frac{\text { IgG anti-prM-E }}{(+) \quad \text { Total horse sera }}$

\section{IgG anti-WARF4}

\begin{tabular}{lrrr}
$(+)$ & $5^{\text {a }}$ & 1 & 6 \\
$(-)$ & 10 & 27 & 37 \\
Total horse sera & 15 & 28 & 43 \\
\hline
\end{tabular}

${ }^{a}$ For statistical analysis, results were analyzed by a $2 \times 2$ contingency table. A significant association was found between antibodies to prM-E proteins and WARF4 protein $(\mathrm{p}<0.015)$.

analyzing its reactivity with 43 horse serum samples by Western blotting. Among the others, 15 horse sera showed antibodies against prM-E WNV proteins, while 28 lacked reactivity to the same proteins. Criteria of positivity to WARF4 recombinant protein was the appearance of an immunoreactive band in the WARF4 sample, co-migrating with the one visualized by the anti-his antibody and not in the bacterial cell extract used as negative control. Two proteins in the WARF4 sample were detected in several horse serum samples as well by the anti-his antibody (Fig. 2). The lower molecular weight protein might represent a degradation product of the full length WARF4 recombinant protein. Overall, we detected serum antibodies to WARF4 recombinant purified protein in 5 out of 15 horses which simultaneously displayed antibodies against the prM-E proteins. Serum antibodies were also detected in one of 28 horses testing negative for antibodies to WNV prM-E proteins. To demonstrate the specificity of the humoral response to WARF4, a competition assay was performed (Fig. 3). When using the highest WARF4 protein concentration as competitor, the serum reactivity disappeared thus confirming the presence of specific $\mathrm{IgG}$ for the WARF4 protein. A significant association was found between the presence of antibodies to prM-E and WARF4 proteins in horse serum $(\mathrm{p}<0.015)$ (Table I).

\section{Discussion}

West Nile virus, which has become endemic in North America, is a positive-sense RNA neurotropic virus $(10,11,17)$. The WNV genome encodes 3 structural (capsid, premembrane/ membrane and envelope) and 7 nonstructural proteins $(10,11,17)$. Neutralizing antibodies are essential for the control of the WNV infection $(10,11,17)$. It was demonstrated that MHC molecules can also present peptides encoded in alternate translational reading frames (21). The general importance of out of frame proteins was highlighted for their ability to enhance immunity responses (21). Furthermore, it was demonstrated that these alternative products may have a critical role in the pathogenesis of viral infections $(22,23)$. The WNV genome has been completely sequenced, and our computer-assisted analysis indicates the presence of 6 ARFs. Among the others, alignment analysis shows that the ARF4 (entitled West Nile Alternative Reading 
Frame 4, WARF4), represents a unique feature of lineage I WNV genomes, that are often associated with clinical symptoms. In order to prove the actual existence of the WARF4 protein, we produced a prokaryotic recombinant source of WARF4 and verified its immunogenicity in vivo by analyzing its reactivity with 43 horse sera by Western blotting. The horse sera were also characterized for their reactivity to well known premembrane and envelope (prM-E) WNV proteins by a commercial ELISA kit.

Our results demonstrated a differential reactivity of the horse sera. Five serum samples displayed antibodies for both WARF4 and prM-E WNV proteins; 10 sera, testing positive for prM-E antibodies were WARF4 negative. Conversely, one serum sample lacking immunoreactivity to prM-E appeared, instead, reactive to the WARF4 recombinant protein. Our results indicate a statistically significant association between the presence of antibodies to prM-E proteins and WARF4 in the horse sera $(\mathrm{p}<0.015)$ (Table I). The reactivity of horse sera to prM-E protein but not to WARF4 might be related to the ARF4 expression level. Indeed, ARF proteins are reported to be usually less expressed compared to ORF proteins (24). The fact that a single horse had specific antibodies to the WARF4 protein but not to the prM-E proteins might suggest cross-reactivity of antibodies with cocirculating flavivirus protein (25) or a different sensitivity of the assay used for testing the presence of antibodies.

We have not yet investigated the translation mechanisms of this novel encoded protein. A cap-dependent ribosomal scanning process is involved in $\mathrm{WN}$ virus translation, although a different strategy was reported for other flaviridae (26). Since the WARF4 gene lacks an AUG codon, a potential alternative translation mechanism could take into account the ribosomal frame shift; nevertheless other non-canonical translation mechanisms cannot be excluded. At this stage it is not possible to speculate on the function of this novel protein. Furthermore, it must be highlighted that the presence of antibodies to the WARF4 protein needs to be observed in humans infected by WNV. Overall, our findings provide the evidence of an antibody response to the WARF4 protein in the serum of horse testing positive for antibodies to prM-E proteins, thus indicating that this antigen might be a potential new tool for further characterization of the immune response of WNV infections in humans as well. However, further studies are necessary to show that the WARF4 protein is produced in the context of WNV infection. On the other hand, it will be intriguing to investigate whether a potential association exists, between the evidence of a WARF4 specific immune response and the outcome of the human WNV disease. In this context, it is also suggestive that lineage II WNV genomes lacking WARF4 are not associated to human clinical disease.

\section{References}

1. Smithburn JS, Hughes TP, Burke AW and Paul JH: A neurotropic virus isolated from the blood of a native of Uganda. Am J Trop Med Hyg 20: 471-492, 1970.

2. Monath TP: Flaviviruses. In: Virology. Fields BN and Knipe DM (eds) Raven Press, New York pp763-814, 1990.

3. Marfin AA and Gubler DJ: West Nile encephalitis: an emerging disease in the United States. Clin Infect Dis 33: 1713-1719, 2001.
4. From the Centers for Disease Control and Prevention. West Nile virus activity-United States, August 29-September 4, 2002. JAMA 288: 1465, 2002.

5. Lanciotti RS, Ebel GD, Deubel V, et al: Complete genome sequences and phylogenetic analysis of West Nile virus strains isolated from the United States, Europe and the Middle East. Virology 298: 96-105, 2002.

6. Beasley DW, Li L, Suderman MT and Barrett AD: Mouse neuroinvasive phenotype of West Nile virus strains varies depending upon virus genotype. Virology 296: 17-23, 2002.

7. Keller BC, Fredericksen BL, Samuel MA, Mock RE, Mason PW, Diamond MS and Gale M Jr: Resistance to alpha/beta interferon is a determinant of West Nile virus replication fitness and virulence. J Virol 80: 9424-9434, 2006.

8. Samuel MA and Diamond MS: Pathogenesis of West Nile virus infection: a balance between virulence, innate and adaptive immunity and viral evasion. J Virol 80: 9349-9360, 2006.

9. Evans JD and Seeger C: Differential effects of mutations in NS4B on West Nile virus replication and inhibition of interferon signaling. J Virol 81: 11809-11816, 2007.

10. Rios M, Daniel S, Dayton AI, Wood O, et al: In vitro evaluation of the protective role of human antibodies to West Nile virus (WNV) produced during natural WNV infection. J Infect Dis, 2008 Sep 4. (Epub ahead of print).

11. Oliphant T, Nybakken GE, Austin SK, et al: Induction of epitope-specific neutralizing antibodies against West Nile virus. J Virol 81: 11828-11839, 2007.

12. Sanger F, Air GM, Barrell BG, et al: Nucleotide sequence of bacteriophage phi X174 DNA. Nature 265: 687-695, 1977.

13. Baranov PV, Henderson CM, Anderson CB, Gesteland RF, Atkins JF and Howard MT: Programmed ribosomal frameshifting in decoding the SARS-CoV genome. Virology 332: 498-510, 2005.

14. Gurvich OL, Baranov PV, Zhou J, Hammer AW, Gesteland RF and Atkins JF: Sequences that direct significant levels of frameshifting are frequent in coding regions of Escherichia coli. EMBO J 22: 5941-5950, 2003.

15. Matsufuji S, Matsufuji T, Miyazaki Y, Murakami Y, Atkins JF, Gesteland RF and Hayashi S: Autoregulatory frameshifting in decoding mammalian ornithine decarboxylase antizyme. Cell 80: 51-60, 1995.

16. Walewski JL, Keller TR, Stump DD and Branch AD: Evidence for a new hepatitis $C$ virus antigen encoded in an overlapping reading frame. RNA 7: 710-721, 2001.

17. Trevejo RT and Eidson M: Zoonosis update: West Nile virus. J Am Vet Med Assoc 232: 1302-1309, 2008.

18. Bei R, Masuelli L, Trono P, et al: The ribosomal P0 protein induces a spontaneous immune response in patients with head and neck advanced stage carcinoma that is not dependent on its overexpression in carcinomas. Int J Oncol 31: 1301-1308, 2007.

19. Masuelli L, Trono P, Marzocchella L, et al: Intercalated disk remodeling in $\delta$-sarcoglycan-deficient hamsters fed with an $\alpha$ linolenic acid-enriched diet. Int J Mol Med 21: 41-48, 2008.

20. Masuelli L, Focaccetti C, Cereda V, et al: Gene-specific inhibition of breast carcinoma in BALB-neuT mice by active immunization with rat $\mathrm{Neu}$ or human ErbB receptors. Int $\mathrm{J}$ Oncol 30: 381-392, 2007.

21. Malarkannan S, Horng T, Shih PP, Schwab S and Shastri N: Presentation of out-of-frame peptide/MHC class I complexes by a novel translation initiation mechanism. Immunity 10: 681-690, 1999.

22. Ma HC, Lin TW, Li H, et al: Hepatitis $\mathrm{C}$ virus ARFP/F protein interacts with cellular MM-1 protein and enhances the gene trans-activation activity of c-Myc. J Biomed Sci 15: 417-425, 2008.

23. Wolf M, Dimitrova M, Baumert TF and Schuster C: The major form of hepatitis $C$ virus alternate reading frame protein is suppressed by core protein expression. Nucleic Acids Res 36: 3054-3064, 2008.

24. Baril M, Dulude D, Gendron K, Lemay G and Brakier-Gingras L: Efficiency of a programmed-1 ribosomal frameshift in the different subtypes of the human immunodeficiency virus type 1 group M. RNA 9: 1246-1253, 2003.

25. Crill WD, Trainor NB and Chang GJ: A detailed mutagenesis study of flavivirus cross-reactive epitopes using West Nile virus-like particles. J Gen Virol 88: 1169-1174, 2007.

26. Edgil D, Polacek C and Harris E: Dengue virus utilizes a novel strategy for translation initiation when cap-dependent translation is inhibited. J Virol 80: 2976-2986, 2006. 